\title{
Searches for nonresonant new phenomena in final states with leptons and photons at the CMS Experiment
}

\author{
Kyungwook Nam ${ }^{* \dagger}$ \\ Seoul National University \\ E-mail: kyungwook.namecern.ch
}

Many new physics models, e.g., compositeness, see-saw, and extra dimensions models, are expected to manifest themselves in the final states with leptons and photons. This talk presents searches for new nonresonant phenomena in the final states that include leptons and photons, focusing on the recent results obtained using data collected at Run-II of the LHC.

The 39th International Conference on High Energy Physics (ICHEP2018)

4-11 July, 2018

Seoul, Korea

*Speaker.

${ }^{\dagger}$ On behalf of the CMS Collaboration 


\section{Introduction}

Various new physics models such as compositeness and extra dimensions models are expected to manifest themselves in final states with leptons and photons. This paper presents searches for new nonresonant phenomena in final states that include leptons and photons, based on data from proton-proton collisions at a center-of-mass energy of $13 \mathrm{TeV}$ at the LHC in 2016, corresponding to an integrated luminosity of $35.9 \mathrm{fb}^{-1}$, and collected with the CMS detector [1].

\section{Excited leptons in $l l \gamma$ final states}

A search is presented for excited leptons in $l l \gamma$ final states ( $l$ refers to $e$ and $\mu$ ) [2]. 95\% confidence level (CL) upper limits are set on the product of the production cross section and branching fraction, and the corresponding lower limits on the compositeness scale $\Lambda$ are also set, as shown in Fig. 1. Excited electrons and muons are excluded for masses below 3.9 and $3.8 \mathrm{TeV}$, respectively, in the case $m_{l^{*}}=\Lambda$. The best observed limit on $\Lambda$ is obtained with an excited lepton mass of around 1.0 TeV, excluding values below $25 \mathrm{TeV}$ for both excited electrons and muons. These are the most stringent limits on excited leptons to date.
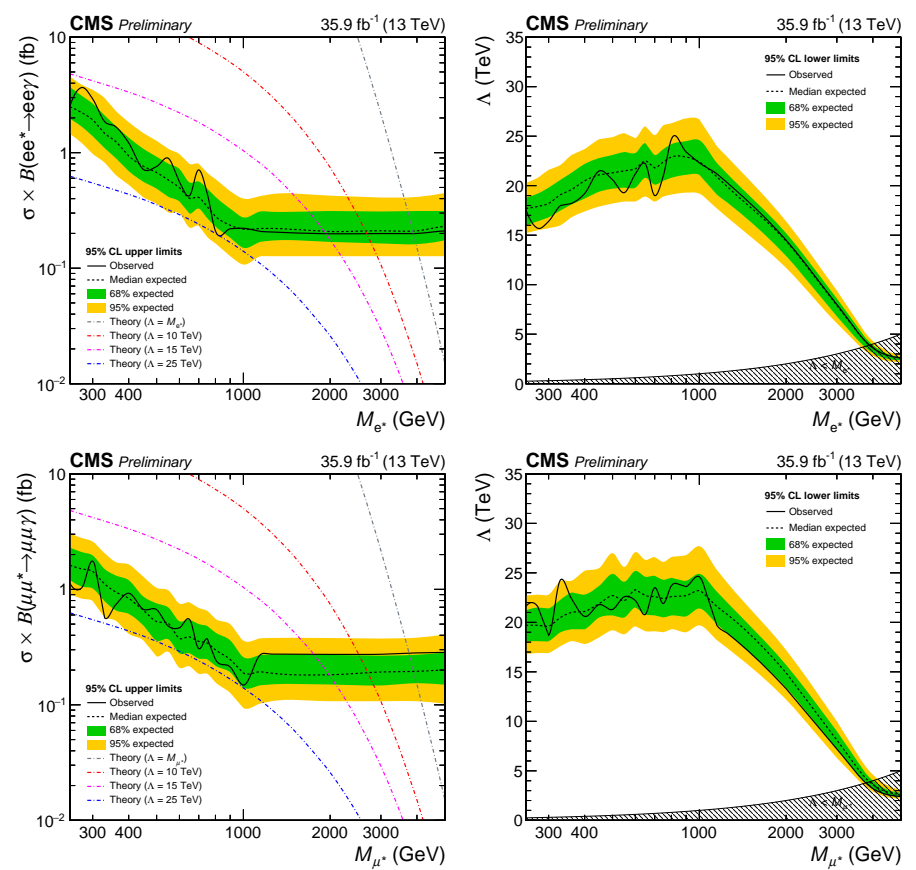

Figure 1: Limits on the product of the production cross section and branching fraction (left column) and lower limits on $\Lambda$ (right column) as a function of signal mass for excited electrons (upper row) and muons (lower row) [2].

\section{Monophoton}

A search for new physics in final states including a photon and missing transverse momentum is conducted [3]. The results are interpreted in the context of models with extra spatial dimensions, 
and limits on new physics parameters are set at 95\% CL, as shown in Fig. 2. Values of the effective Planck scale $M_{D}$ up to $2.85-2.90 \mathrm{TeV}$ are excluded for between 3 and 6 extra spatial dimensions.
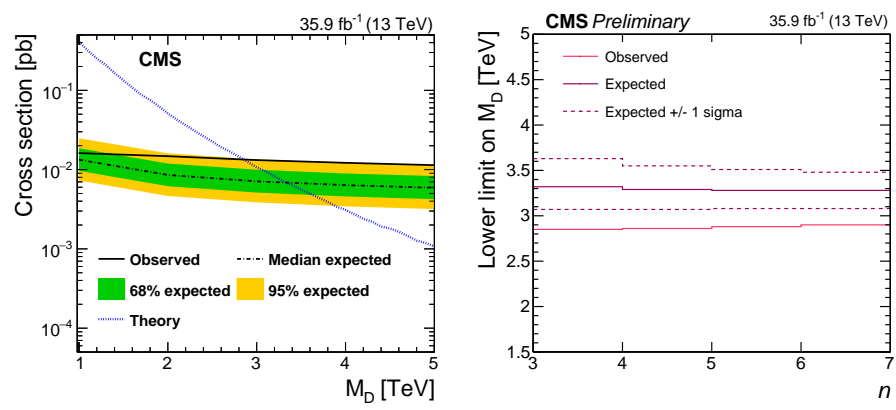

Figure 2: Limits on the ADD graviton production cross section as a function of $M_{D}$, for 3 extra dimensions (left), and lower limit on $M_{D}$ as a function of $\mathrm{n}$, the number of ADD extra dimensions (right) [3].

\section{MonoZ(II)}

A search is presented for new physics in events with a $\mathrm{Z}$ boson associated with large missing transverse momentum at the LHC [4]. The results are interpreted in terms of modelsl of unparticle production and large extra spatial dimensions, as shown in Fig. 3. No significant deviations from the SM background expectations are observed, and limits are calculated on the model parameters, significantly extending the previous results in this channel.
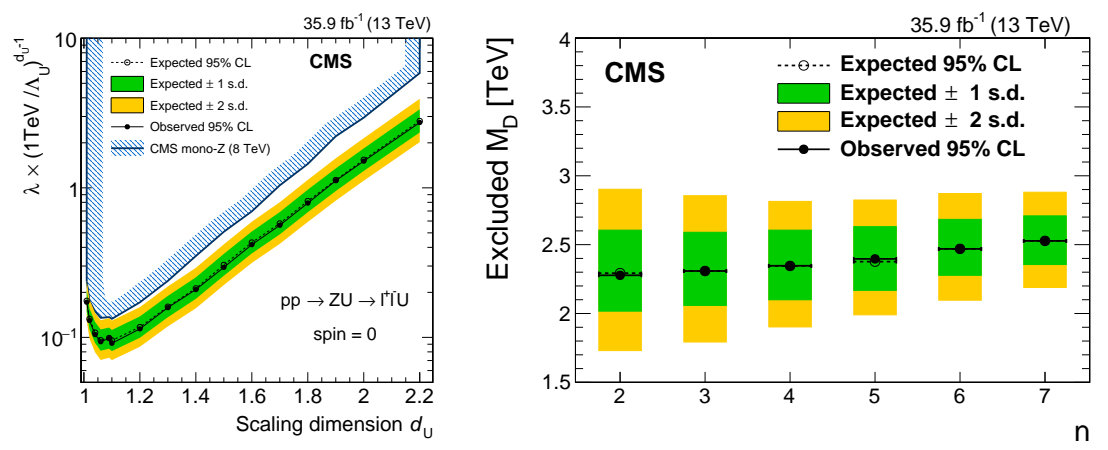

Figure 3: Upper limits on the Wilson coefficient of the unparticle-quark coupling operator (left) and exclusion limits on $M_{D}$ for different values of $\mathrm{n}$, the number of ADD extra dimensions (right) [4].

\section{Nonresonant diphoton}

A search for physics beyond the standard model is performed using a sample of high-mass diphoton events [5]. For the large extra-dimensional model of Arkani-Hamed, Dimopoulos, and Dvali, lower limits are set on the string mass scale $M_{S}$ ranging from 5.6 to $9.7 \mathrm{TeV}$, depending on the model parameters, as summarized in Table. 1. The first exclusion limits are set in the twodimensional parameter space of a continuum clockwork model, as shown in Fig. 4. 
Table 1: Exclusion lower limits obtained on the mass scale (in units of $\mathrm{TeV}$ ) for various conventions used in the calculation of the ADD large extra dimensional scenario [5].

\begin{tabular}{cccccccccc} 
Signal & \multirow{2}{*}{ GRW } & \multicolumn{2}{c}{ Hewett } & \multicolumn{7}{c}{ HLZ } \\
& & negative & positive & $n_{E D}=2$ & $n_{E D}=3$ & $n_{E D}=4$ & $n_{E D}=5$ & $n_{E D}=6$ & $n_{E D}=7$ \\
\hline Expected & $7.1_{-0.5}^{+0.7}$ & $5.5_{-0.3}^{+0.1}$ & $6.3_{-0.4}^{+0.6}$ & $8.4_{-1.1}^{+1.3}$ & $8.4_{-0.6}^{+0.8}$ & $7.1_{-0.5}^{+0.7}$ & $6.4_{-0.5}^{+0.6}$ & $6.0_{-0.4}^{+0.6}$ & $5.6_{-0.4}^{+0.6}$ \\
Observed & 7.8 & 5.6 & 7.0 & 9.7 & 9.3 & 7.8 & 7.0 & 6.6 & 6.2
\end{tabular}

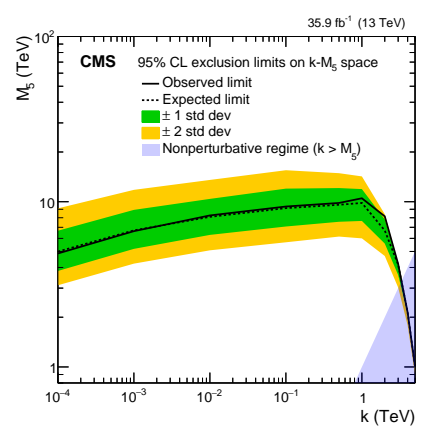

Figure 4: Limits for the continuous graviton model in the clockwork framework over the k-M5 parameter space [5].

\section{Acknowledgments}

Kyungwook Nam is supported in part by the National Research Foundation of Korea (NRF) funded by the Korea government (NRF-2018R1A1A1A05077514 and NRF-2015R1A4A1042542).

\section{References}

[1] CMS Collaboration, "The CMS experiment at the CERN LHC", JINST 3 (2008) S08004.

[2] CMS Collaboration, "Search for excited leptons in the $l l \gamma$ final state in proton-proton collisions at $\sqrt{s}=13$ TeV", arXiv:1811.03052, Submitted to JHEP.

[3] CMS Collaboration, "Search for new physics in final states with a single photon and missing transverse momentum in proton-proton collisions at $\sqrt{s}=13 \mathrm{TeV}$ ", arXiv:1810.00196, Submitted to JHEP.

[4] CMS Collaboration, "Search for new physics in events with a leptonically decaying Z boson and a large transverse momentum imbalance in proton-proton collisions at $\sqrt{s}=13 \mathrm{TeV}$ ", Eur. Phys. J. C 78 (2018) 291.

[5] CMS Collaboration, "Search for physics beyond the standard model in high-mass diphoton events from proton-proton collisions at $\sqrt{s}=13 \mathrm{TeV}$ ", arXiv:1809.00327, Submitted to Phys. Rev. D. 